\title{
Gradhiva
}

GRADHI

Revue d'anthropologie et d'histoire des arts

$10 \mid 2009$

Présence africaine

\section{Pap Ndiaye, La Condition noire. Essai sur une minorité française}

Paris, Calmann-Lévy, 2008

Dominic Thomas

\section{OpenEdition}

\section{Journals}

Édition électronique

URL : http://journals.openedition.org/gradhiva/1594

DOI : 10.4000/gradhiva. 1594

ISSN : 1760-849X

\section{Éditeur}

Musée du quai Branly Jacques Chirac

Édition imprimée

Date de publication : 4 novembre 2009

Pagination : 218-219

ISBN : 978-2-35744-012-8

ISSN : 0764-8928

\section{Référence électronique}

Dominic Thomas, «Pap Ndiaye, La Condition noire. Essai sur une minorité française », Gradhiva [En

ligne], 10 | 2009, mis en ligne le 03 février 2010, consulté le 21 septembre 2020. URL : http:// journals.openedition.org/gradhiva/1594; DOI : https://doi.org/10.4000/gradhiva.1594

Ce document a été généré automatiquement le 21 septembre 2020.

(C) musée du quai Branly 


\section{Pap Ndiaye, La Condition noire. Essai sur une minorité française}

Paris, Calmann-Lévy, 2008

Dominic Thomas

\section{RÉFÉRENCE}

Pap Ndiaye, La Condition noire. Essai sur une minorité française, Paris, Calmann-Lévy, 2008

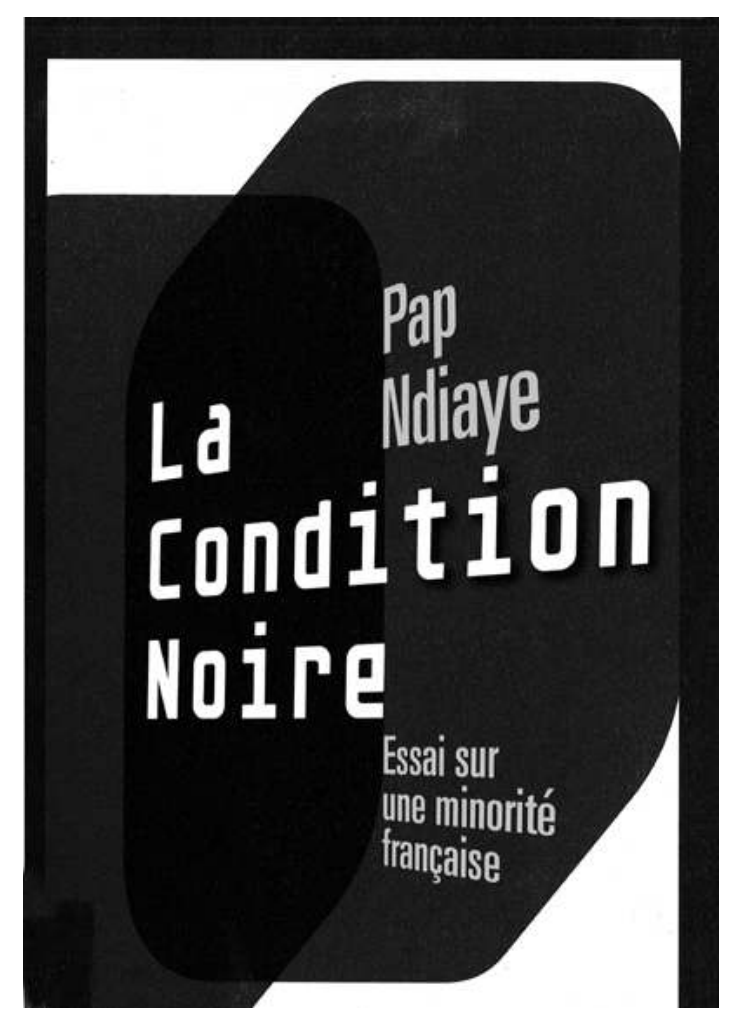


1 Si de nombreux facteurs ont contribué à donner à la question noire une réelle importance dans la France d'aujourd'hui, les spectaculaires émeutes qui se sont produites dans les banlieues françaises en 2005 ont particulièrement mis en lumière l'ampleur des inégalités sociales et l'impact profond qu'elles ont sur les minorités visibles. Jusqu'à présent, les discussions sur les minorités ethniques françaises s'étaient concentrées sur les disparités entre les problèmes culturels et sociaux de ces communautés et leurs exigences, des exigences jugées incompatibles avec les valeurs républicaines, considérées comme autant d'obstacles à l'intégration et de limites à l'assimilation, et donc impossibles à incorporer aux normes d'une francité vague mais soutenue par les autorités et les médias. Bien que la catégorisation raciale ait effectivement été propulsée au premier plan de l'actualité, rien n'a aujourd'hui changé dans les relations ténues existant entre les minorités visibles et ces mêmes autorités. La question n'est pas nouvelle - c'est en 1969, il y a maintenant quarante ans, que le grand écrivain malien Yambo Ouologuem a publié sa Lettre à la France nègre. La question raciale se trouve donc reformulée selon plusieurs facteurs historiques et sociaux qui s'articulent autour d'un parcours lié à l'esclavage, au colonialisme ainsi qu'à l'époque proprement postcoloniale, époque à laquelle de nouveaux éléments sont venus compliquer les paramètres de la discussion, étant donné l'absence de toute homogénéité au sein de la population noire aujourd'hui en France. «Depuis une dizaine d'années, selon Ndiaye, les Noirs vivant en France, de nationalité française ou pas, font entendre leur voix et apparaissent visiblement dans l'espace public national » (p. 18). Ce sont ces questions qui forment le propos de Ndiaye dans La Condition noire, un ouvrage qui incite à la réflexion notamment du fait de sa «dimension francoaméricaine »(p.25), mêlant les références aux African American studies ainsi qu'aux recherches anglo-saxonnes dans le domaine des cultural studies et des postcolonial studies.

2 Le premier élément à souligner concerne la dimension paradoxale de la question noire. En effet, « en tant que groupe social [les Noirs] sont censés ne pas exister, puisque la République française ne reconnait pas officiellement les minorités, et ne les compte pas non plus » (p. 17). Le passage d'une invisibilité juridique à une visibilité d'ordre racial, passage qui s'accompagne d'un ensemble de revendications et d'une mobilisation collective, représente donc un défi complexe. L'approche choisie par Pap Ndiaye s'inscrit dans la pensée d'une "politique minoritaire » afin de se "concentrer sur les phénomènes par lesquels des groupes spécifiques, les "minorités", se trouvent discriminés, c'est-à-dire objets de traitements différenciés fondés sur des critères illégitimes [...] un groupe de personnes ayant en partage, nolens volens, l'expérience sociale d'être généralement considérées comme noires » (p. 21-24). Alors qu'aux yeux de certains chercheurs la perte de l'invisibilité raciale risque d'aboutir à une définition essentialiste des groupes, Ndiaye insiste au contraire sur l'existence d'une «logique minoritaire " (p. 368) plutôt que "communautaire" " (p.368), «relative à une expérience sociale concrète " (369), à savoir comment être simultanément français et noir. Cette prise de conscience du caractère partagé de l'expérience discriminatoire pourrait alors motiver l'idée d'une organisation sociale destinée à mettre fin à ce traitement plutôt que d'alimenter un objectif communautariste. À l'évidence, ces contradictions se retrouvent dans les choix du président Nicolas Sarkozy, qui a nommé dans son premier gouvernement un échantillon de minorités visibles. Cependant, suite à une campagne électorale fondée sur la stigmatisation de l'étranger et de l'immigré et renforcée par la création dans ce même gouvernement d'un ministère de 
«l'Immigration et de l'Identité nationale », l'appartenance à la France est remise en question par une interprétation limitée de l'histoire et de la démographie. Il devient donc essentiel de repenser la longue histoire de la présence noire en France et la dimension constitutive des relations qui en découlent, tout en insistant sur son aspect symbiotique afin d'approfondir les débats sur l'identité française et de reconsidérer la singularité démographique de cet espace. Prenant pour sujet «les Noirs de France métropolitaine du point de vue de leur sociologie, et de ce qui les affecte sur le plan de leur existence comme individus et comme groupe» (p. 19), La Condition noire de Pap Ndiaye entend contribuer à une mise en forme scientifique "qui paraît désormais urgente» (p. 19).

3 Organisé autour d'une bibliographie destinée à fournir une perspective à la fois historique et sociologique, d'entretiens sur le terrain et d'une étude des associations noires en/de de France, le livre est divisé en six chapitres. La première partie s'interroge sur le terme "Noir», expliquant « en quoi la notion de minorité est utile pour penser la condition noire» (p. 25), examinant «les distinctions et hiérarchies sociales qui existent depuis l'esclavage entre Noirs selon leur degré de mélanine " (p. 25), et offrant « une histoire synthétique des populations noires de France depuis le XVIII ${ }^{\mathrm{e}}$ siècle » (p. 26). Cette mise en contexte est d'autant plus utile qu'elle est le plus souvent absente de la plupart des analyses plus récentes sur ces questions. Conscient du fait que la thématique elle-même est aujourd'hui fortement politisée, l'auteur, qu'il le veuille ou non, engage la deuxième partie de son livre dans une polémique qui «explore une série de questions historiques [...] et sociologiques [...] ainsi que les arguments antiracistes utilisés par les Noirs» (p. 27). Dans le même temps, il «rend compte du déplacement de la lutte antiraciste vers la politique antidiscriminatoire", "plaide pour l'utilisation de techniques statistiques afin d'établir la discrimination comme un fait social» (p. 27), et, en guise de conclusion, « propose une solidarité noire fondée sur l'identité "fine" des expériences et des intérêts communs, plutôt que sur l'identité "épaisse" des origines et des cultures » (p. 27), tout en évaluant les formes de solidarité entre Noirs dans la France d'aujourd'hui.

4 En 2008, le gouvernement français a commandé deux rapports : celui du comité de réflexion sur le préambule de la Constitution sous la direction de Simone Veil, et $L a$ Diversité : rapport à la ministre de l'Enseignement supérieur et de la Recherche, dirigé par le sociologue Michel Wieviorka. Confirmant son intérêt pour la question de la diversité dans la France d'aujourd'hui, le président Sarkozy a nommé Yazid Sabeg commissaire à la Diversité, qui s'est chargé de mettre en place en mars 2009 le Comité pour la mesure et l'évaluation des discriminations (Comedd). Mais, dans le même temps, un groupe de chercheurs établissait la Commission alternative de réflexion sur les "statistiques ethniques » et les « discriminations » (Carsed). L'originalité et l'importance du livre de Ndiaye se situent précisément dans son approche de ces questions. Dans un premier temps, Ndiaye met en évidence l'évolution sémantique et la transformation de la lutte contre le racisme en une lutte contre les discriminations, phénomène qui coïncide avec la mobilisation associative. L'auteur pointe toutefois une distinction importante en ayant recours au cadre américain : "L'idéal d'un monde déracialisé suppose, du point de vue américain, que l'on ne ferme pas les yeux sur les différences actuelles entre Noirs et Blancs [...] il convient non point de nier absolument toute différence entre eux, mais au contraire de les mesurer pour les réduire à toutes fins utiles" (p. 252). L'argumentation qui suit en faveur de la mesure statistique répond à une logique claire et convaincante: "Pour fonder objectivement le caractère discriminatoire de 
l'inégalité de traitement, la comparaison est indispensable, puisqu'elle permet d'établir des torts éventuels » (p. 278), car, si « les personnes minorées [...] peinent en France à faire valoir leurs difficultés, c'est aussi faute de l'équipement statistique sur lequel elles pourraient s'appuyer pour s'organiser et revendiquer» (p. 285). Pour Ndiaye, par opposition par exemple à la position du Carsed, et en s'appuyant sur une analyse approfondie de plusieurs modèles comparatifs, comme l'affirmative action aux ÉtatsUnis et le Race Relations Act au Royaume-Uni, «Il est donc prioritairement question de factualiser les discriminations fondées sur des critères ethno-raciaux, qu'il s'agit bien de nommer si on veut les combattre, plutôt que de se voiler la face dans une posture pseudo républicaine » (p. 285).

Ndiaye n'hésite donc pas à s'engager dans une polémique complexe et actuelle. Reconnaissant l'attention limitée qu'ont reçue ces questions en France de la part des universitaires, l'auteur s'efforce de proposer «des interprétations mais aussi des hypothèses et des pistes de recherches futures à un champ d'étude qui pourrait être celui des black studies à la française» (p.19). Reste que ce livre n'est pas le premier sur le sujet : «N'oublions pas qu'il existe certes de bonnes études sur les familles africaines, les immigrés africains, les migrants antillais et réunionnais, les agents publics antillais, les tirailleurs sénégalais, etc.» (p. 18). Si La Condition noire s'annonce bien comme un travail incontournable pour des "black studies à la française ", il n'en est pas le texte fondateur. En effet, une longue liste de chercheurs en France et ailleurs - Achille Mbembe, Étienne Balibar, Éric Fassin, Didier Fassin, Édouard Glissant, Françoise Vergès, Pascal Blanchard, Nicolas Bancel et Sandrine Lemaire - ont démontré que la présence noire en France s'inscrit dans un contexte historique pluridimensionnel et se caractérise par une diaspora marquée par la globalité. En cela, ils ont aidé, comme Ndiaye aujourd'hui, à repenser la longue histoire française, sans masquer la dimension fondatrice de l'expérience historique collective, et contribué à accorder une légitimité à un champ de recherche non pas en le créant, mais plutôt en l'enrichissant à travers de nouvelles approches, de nouvelles connexions, de nouvelles évaluations et de nouvelles interprétations.

\section{AUTEURS}

\section{DOMINIC THOMAS}

dominict@humnet.ucla.edu 